\title{
On-chip Biosensing with high-Q Amorphous Silicon Microdisk Resonators
}

\author{
T. Lipka, J. Amthor, H.K. Trieu, J. Müller \\ Institute of Micro System Technology, Hamburg University of Technology, \\ Eißendorfer Str. 42, 21073 Hamburg, Germany \\ timo.lipka@tuhh.de
}

\begin{abstract}
:
Low-loss hydrogenated amorphous silicon (a-Si:H) microdisk resonators which are employed as evanescent field refractive index sensors are presented. The resonances of the whispering gallery modes exhibit extinction ratios of $>25 \mathrm{~dB}$ and $\mathrm{Q}$-factors $\sim 10^{4}$ in aqueous solutions. A sensitivity as high as $>400 \mathrm{~nm} / \mathrm{RIU}$ was measured for $\mathrm{NaCl}$-solved in DI-water. The resonators intrinsic limit of detection was calculated to be $L O D=3.3 \times 10^{-4}$ and the minimum detectable amount of $\mathrm{NaCl}$ was experimentally determined to be $0.0375 \%$. The measurements of the photonic evanescent field microdisk sensors prove that hydrogenated amorphous silicon photonic resonators can be exploited for label-free lab-onchip sensing applications.
\end{abstract}

Keywords: amorphous silicon, microdisk resonator, label-free, lab-on-chip, sensing.

\begin{abstract}
Intoduction
Photonic sensors which are built up with high refractive index photonic microring or microdisk resonators are very attractive for optical sensing. In particular, silicon is a promising platform to fabricate photonic lab-on-chip sensors for chemical, medical and bio-sensing applications. This is due to the potential mass fabrication capabilities at low-costs and the possibility to fabricate large sensor arrays on a common substrate with ultra-compact sized resonators that offer high sensitivities [1].
\end{abstract}

Hydrogenated amorphous silicon (a-Si:H) which is deposited by plasma enhanced chemical vapor deposition (PECVD) is an alternative material to $\mathrm{SOI}$ as it provides a high refractive index and low-loss material for near-infrared photonics, both comparable to crystalline silicon $[2,3]$. However, compared to $\mathrm{c}-\mathrm{Si}, \mathrm{a}-\mathrm{Si} \mathrm{H}$ offers more flexibility in the fabrication process as it can be deposited at relatively low temperatures $\left(<300^{\circ}\right)$ and therefore allows to use glass or plastic materials as substrates. This facilitates a low-cost fabrication and enables a more flexible processing platform, e.g. the resonator is fabricated in a vertically stacked configuration and combined with micro-fluidics.

In this paper we present CMOS-compatible amorphous silicon microdisk resonators that exhibit loaded Q-factors $\sim 10^{4}$ for the transversal magnetic mode ( $q-T M)$ and were employed as evanescent field refractive index sensors. The low mode volume of the whispering gallery modes and the high $Q$ factors which can be achieved with photonic disk resonators make them well suited for lab-on-chip sensing applications.

The photonic systems were patterned with electron beam lithography and structured with inductively coupled plasma etching, resulting in $480 \times 200 \mathrm{~nm}$ photonic wire waveguides and disk resonators with $10 \mu \mathrm{m}$ radius. The resonance peaks of critically coupled disk resonators showed extinction ratios up to $30 \mathrm{~dB}$ with an aqueous top cladding of deionized water (DIwater) and a sensitivity of $460 \mathrm{~nm} / \mathrm{RIU}$ was determined from homogeneous sensing experiments with different $\mathrm{NaCl}$ concentrations.

\section{Sensing Principle of Evanescent Field Microdisk Resonators}

In principle, different kind of transparent optical materials like e.g. nitrides, polymers, and glasses can be employed to fabricate photonic resonators for lab-on-chip sensing applications [4]. However, there are advantages of employing a high refractive index material like silicon. The main reason is the compatibility of advanced CMOS-technology with welldeveloped infrastructure and longtime process experience which will allow sharing existing facilities with electronics, or to combine electronic and photonic systems on the same 
substrate. Furthermore, the high index contrast (HIC) between waveguide core and cladding materials (e.g. Si and $\mathrm{SiO}_{2}$ with $\Delta \mathrm{n}>2$ ) permits a high integration density and thus photonic systems with low footprint so that hundreds of sensor arrays with multiple channels can be cost-effectively fabricated on a single wafer. This makes the silicon platform interesting for applications like point-of-care diagnostics.

However, the HIC is just as much beneficial in terms of the sensor sensitivity. The strong mode confinement in single-mode photonic wire waveguides with typical dimensions of $500 \times 200 \mathrm{~nm}$ allow for small bending radii $r<5 \mu \mathrm{m}$ with negligible losses of $\sim 0.01 \mathrm{~dB}$ per full $5 \mu \mathrm{m}$ circle. As a result of that, a large free spectral range of $\sim 18 \mathrm{~nm}$ can be achieved with $5 \mu \mathrm{m}$ ring resonators, which facilitates a distinct detection of large analyte changes. The tight light guidance implies that there is a strong electric field discontinuity at the low and high refractive index layer interfaces and thus the evanescent field can effectively interact with the sensing medium which covers the photonic sensor.

The typical photonic wires guide two fundamental modes, which are the transversal electric (qTE) and the transversal magnetic mode (qTM). The modes of a $480 \times 200 \mathrm{~nm}$

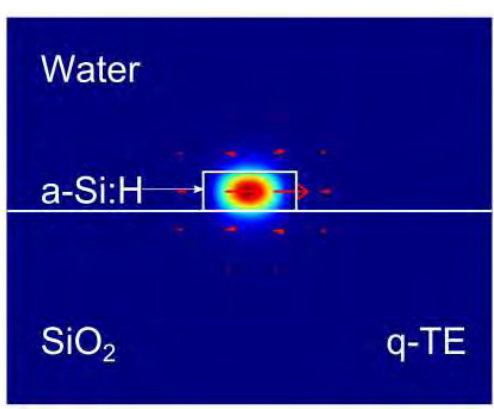

(a)

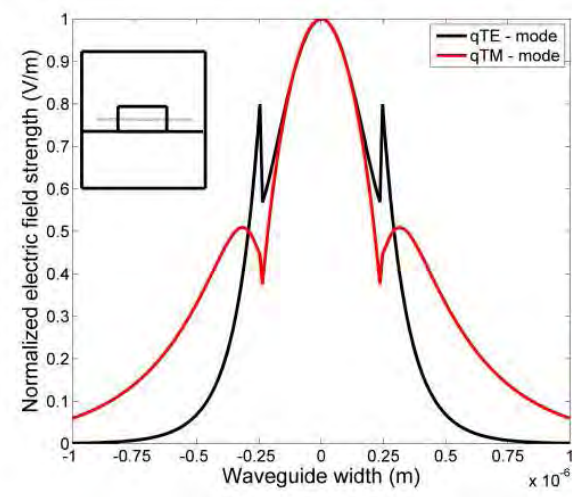

(c) dimensioned photonic wire were simulated at $1550 \mathrm{~nm}$ wavelength by using a finite element mode solver and the results are presented in Fig. 1 a,b).

For the simulations the bulk refractive indices of the materials were implemented as follows, $n=3.478$ for the amorhous silicon core, $n=1.444$ for the oxide under cladding, and $n=1.31$ for the water top cladding. The effective mode indices and the mode confinement factors, which are a measure of the light guided inside the core, were determined as well and are summarized in table 1). The intensity plots indicate that the qTE-mode is stronger confined compared to the qTM-mode, which decreases the interaction of this mode with the sensing medium. The normalized electric field profiles of both modes which show the evanescent field distribution for a line plot across the photonic waveguides width and height are shown in Fig. $1 \mathrm{c}, \mathrm{d}$ ).

Tab. 1: Effective mode index and confinement factor for both fundamental modes.

\begin{tabular}{|c|c|c|}
\hline Mode & $\begin{array}{c}\text { Effective mode } \\
\text { index }\left(\mathrm{N}_{\text {eff }}\right)\end{array}$ & $\begin{array}{c}\text { Confinement } \\
\text { factor }(\Gamma)\end{array}$ \\
\hline qTE & 2.308 & 0.749 \\
\hline qTM & 1.566 & 0.245 \\
\hline
\end{tabular}

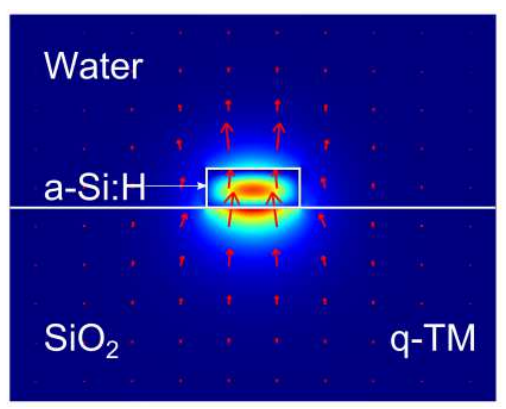

(b)

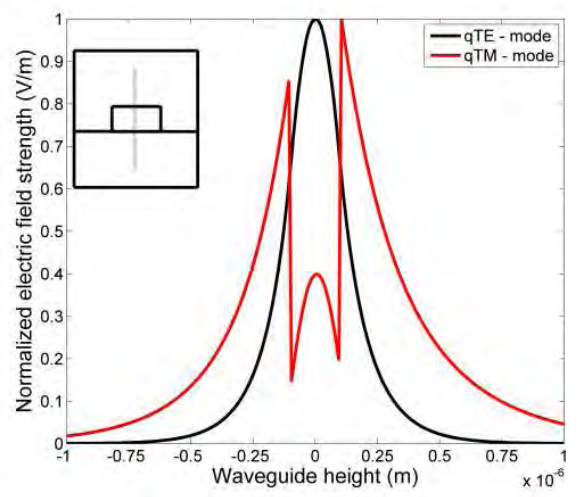

(d)

Fig. 1: Results of FEM simulations: Fundamental qTE (a) and qTM (b) guided mode intensity plots for a photonic waveguide with $480 \times 200 \mathrm{~nm}$ dimensions. The electric field orientation is inset as arrows. Normalized electric field line plots of the fundamental modes for the width (c) and the height (d) of the photonic wires. 
Photonic evanescent field refractive index sensors can be categorized due to different sensing principles as shown in Fig.2 [5]. Surface sensing is a method where selective receptor molecules are immobilized on a functionalized photonic device surface so that only target molecules will selectively stick to the receptors. This way the propagation of the guided mode $\left(\mathrm{N}_{\text {eff }}\right)$ is slightly altered and hence the response of the photonic sensor is a measure of the adsorpted molecule amount. The surface sensing technique is very promising for lab-on-chip or point-of-care biosensing applications.

Compared to that, homogeneous sensing might be employed to measure gases and chemicals. In homogeneous sensing the photonic sensor is covered with a cladding medium, e.g. water or air, and a change of a specific concentration in the medium (e.g. $\mathrm{NaCl}$ ) is detected by the refractive index difference $\Delta n$ of the analyte. For both methods the interrogation of the sensor can be performed with spectral transmission measurements and the signal can be evaluated either by a resonance peak shift, or by an increase in absorption for example induced by gases in the mid-infrared. In this work homogeneous sensing was employed in order to detect the refractive index change by measuring the resonance wavelength shift due to different $\mathrm{NaCl}$ concentrations. The intrinsic sensitivity $\left(\mathrm{S}_{\mathrm{RIU}}\right)$ of the refractive index microdisk sensor can be defined as:

$S_{\text {RIU }}=\frac{\Delta \lambda_{\text {res }}}{\Delta n_{\text {fluid }}}$,

with $\Delta \lambda_{\text {res }}$ as peak shift of a resonant mode that is related to a difference in the fluids refractive index $\Delta \mathrm{n}_{\text {fluid. }}$. The minimum detectable change $\Delta \mathrm{n}_{\min }$ for a given wavelength $\lambda$, the limit of detection (LOD), is calculated as follows:

$$
L O D=\Delta n_{\min }=\frac{\lambda}{Q S},
$$

with $Q$ as quality factor of the resonance peak, which corresponds to the photon lifetime inside the resonator and therefore determines the effective interaction time with the analyte, and is defined as ratio of the resonant peak position and the full width half maximum or $3 \mathrm{~dB}$ bandwidth $\Delta \lambda_{3 \mathrm{~dB}}$ :

$$
Q_{\text {loaded }}=\frac{\lambda_{\text {res }}}{\Delta \lambda_{3 d B}} \text {. }
$$

From the set of eq. (1) - (3) it is evident that a high sensitivity is achieved when there is a strong modal overlap of the guided mode with the sensing medium and that additionally a high $Q$ resonator will enhance the LOD. However, a high Q-factor does not necessarily improve the sensitivity, especially for water based solutions, because water adds substantial propagation loss $\left(\sim 10 \mathrm{~cm}^{-1}\right.$ at $\left.1550 \mathrm{~nm}\right)$ and a high quality factor often results of stronger mode confinement in the resonator core and hence less light can effectively interact with the sensing medium [6].

Based on these considerations the sensing experiments were carried out with microdisk resonators which exhibit moderate $Q$-factors of $\sim 10^{4}$, although we have already fabricated critically coupled disk resonators with higher intrinsic $Q$ of $>10^{5}$ for qTE and $\sim 0.9 \times 10^{6}$ for qTM with amorphous silicon [7]. In order to achieve a strong interaction of the evanescent field with the sensing medium the following experiments were conducted for the qTMmode. The disk radius was chosen to be $r=10 \mu m$, so that a sufficiently large free spectral range (FSR 10nm) for resonances of some modal order was achieved. The coupling gap between disk and waveguide was designed to be $280 \mathrm{~nm}$ for weak coupling.

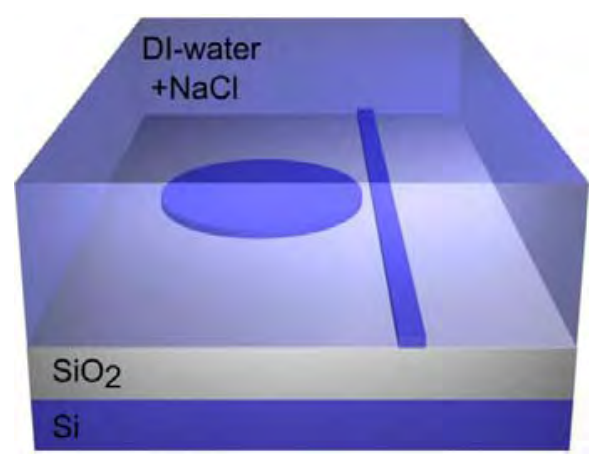

(a)

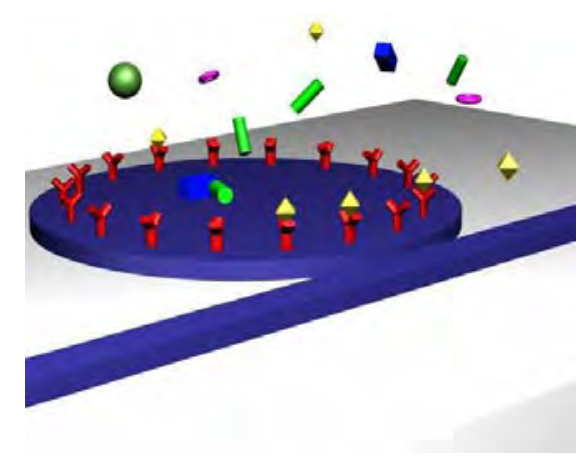

(b)

Fig. 2: (a) Illustration of homogeneous sensing method with a photonic disc resonator covered by an aqueous solution diluted with $\mathrm{NaCl}$. (b) Principle of surface sensing with selective receptor molecules which are immobilized on a functionalized photonic disc resonator surface so that only target molecules selectively stick to the receptors. 


\section{Fabrication and Optical Characterization}

Amorphous silicon was deposited with PECVD on top of thermal oxidized silicon wafers. The photonic microdisks and the supporting waveguides were patterned with electron beam lithography and etched with inductively coupled plasma, which resulted in $480 \times 200 \mathrm{~nm}$ photonic wire waveguides and disk resonators with $10 \mu \mathrm{m}$ in radius as shown in Fig. 3 a). The microdisks were characterized with spectral transmission measurements. With a lensed fiber the probe signal was coupled into the optical waveguides, and a microscope objective was used to collect the light with a fiber-coupled collimator connected to an InGaAs photo detector. Closed-loop controlled piezo-electric alignment stages were used to maximize the coupling to the photonic chip. The NIR-light from the laser was continuously tuned over the desired wavelength range and a $2 \mathrm{GHz}$ oscilloscope that was triggered with the laser output data-logged the transmitted intensity. A thermo-electric controller was used to stabilize the temperature of the chip mount and a constant temperature with variations less than $0.02^{\circ} \mathrm{C}$ was achieved during the measurements.

As a proof of the sensing concept, sodium chloride $(\mathrm{NaCl})$ solved in DI-water was used in order to characterize the proposed evanescent field refractive index sensor. First a reference measurement was performed with pure DIwater, and then liquids with different percentile mass concentrations of $\mathrm{NaCl}$ were dropped onto the disk resonator. The same resonator was used for all measurements and was carefully cleaned with acetone, isopropanol and rinsed with DI-water after each measurement. The measurement results are shown in Fig. 3 b). The graph shows that $1 \%$ of $\mathrm{NaCl}$ causes a resonance wavelength shift of about $0.8 \mathrm{~nm}$. The refractive index change of $1 \% \mathrm{NaCl}$ diluted in DI-water is known to be 0.0017 per mass percent at $\sim 20^{\circ} \mathrm{C}$ [8], which results in an refractive index change of $1.3105-1.32765$ in the given range $(0-10 \%)$. From the measurements the overall sensitivity was determined to be $S_{\text {RIU }} \sim 460 \mathrm{~nm} / \mathrm{RIU}$. The mean $\mathrm{Q}$-factors of the resonances that were used to determine the sensitivity were $\sim 10^{4}$ which results in an intrinsic resonator based limit of detection LOD $=3.3 \times 10^{-4}$.

This LOD is only attributed to the resonator linewidth and can be greatly improved by the readout system (typically by $10^{2}-10^{3}$ ). The detection limit of the current measurement setup was experimentally determined. Therefore a simple experiment was conducted which monitors the evaporation of a thin liquid film with a given starting concentration of $\mathrm{NaCl}$.
When assuming that the evaporation rate of the thin aqueous film is linear over a short time period, the $\mathrm{NaCl}$-concentration and hence the refractive index increase linearly as well. Then the standard deviation from the fit is a measure for the resolution of this experiment.

The results are presented in Fig. $3 \mathrm{c}, \mathrm{d}$ ), where a continuous resonance peak shift towards higher wavelength over time is observed. The peak shift is monitored over 5 minutes taking a measurement each 30 seconds. The overall detected wavelength shift is $\sim 2 \mathrm{~nm}$ which corresponds to an increase in $\mathrm{NaCl}$ percentile mass concentration of $2.5 \%$. The data is fitted and the standard deviation of the data points is $\sim 30 \mathrm{pm}$. From this we conclude an upper detection limit for $\mathrm{NaCl}$ of $0.0375 \%$ which corresponds to a resolution of the bulk refractive index change of the fluid of $\Delta \mathrm{n}_{\min } \sim 6.4 \times 10^{-5}$. It should be noted that this simple experiment is susceptible to errors, e.g. due to a non-uniform $\mathrm{NaCl}$ distribution in the medium, and that theoretically a detection limit $\sim 10^{-6}$ per RIU is possible when the wavelength accuracy is in the $0.5 \mathrm{pm}$ range.

From the current results we suggest that a-Si:H is an attractive photonic material for lab-on-chip sensing applications, especially when combined with micro-fluidic channels which support a well-defined analyte flow rate and if functionalized marker layers with receptors are employed that allow a selective detection of target molecules.

First experiments show that a procedure of piranha etch, amino-silanization and glutaraldehyde functionalization is process-compatible with amorphous silicon material, and early results from the optical characterization of a ring resonator with BSA-protein (bovine serum albumin) adsopted to the ring surface reveal that molecular masses down to a few $\sim \mathrm{fg}$ can be detected. The results will be presented at conference.

\section{Conclusion}

A microdisk sensor based on evanescent field refractive index sensing is fabricated with lowloss hydrogenated amorphous silicon. The whispering gallery modes exhibit $Q$-factors $\sim 10^{4}$ with extinction ratios of $>25 \mathrm{~dB}$ when covered with aqueous solutions. A sensitivity of $>400 \mathrm{~nm} / \mathrm{RIU}$ was determined in homogeneous sensing experiments with different concentrations of $\mathrm{NaCl}$ solved in deionized water. From the measurements the resonator intrinsic limit of detection was calculated to be $3.3 \times 10^{-4}$ and the minimum detectable amount of $\mathrm{NaCl}$ in DI-water was experimentally determined to be $0.0375 \%$. The results prove 


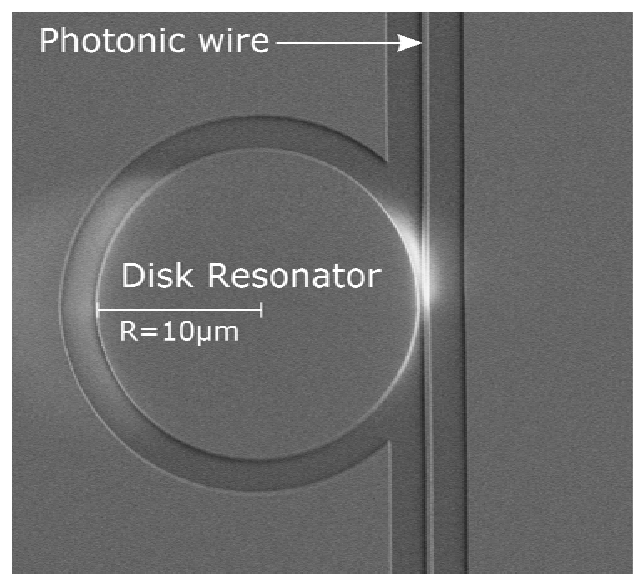

(a)

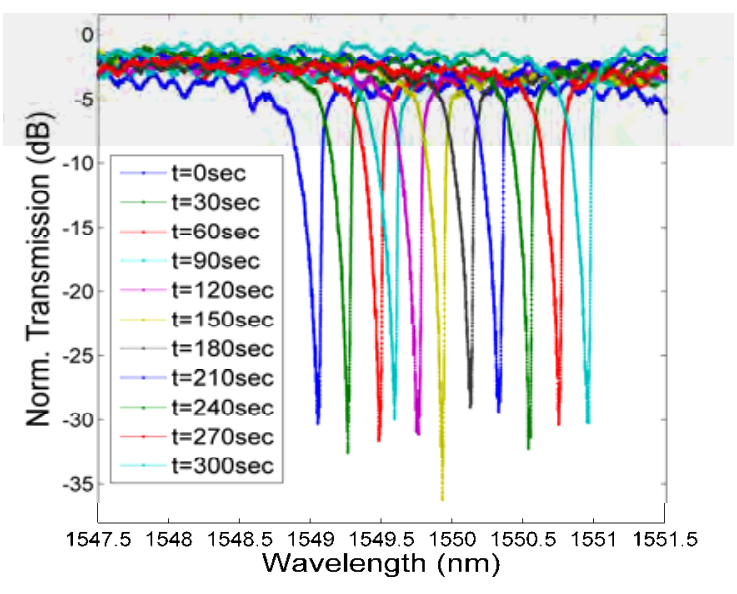

(c)

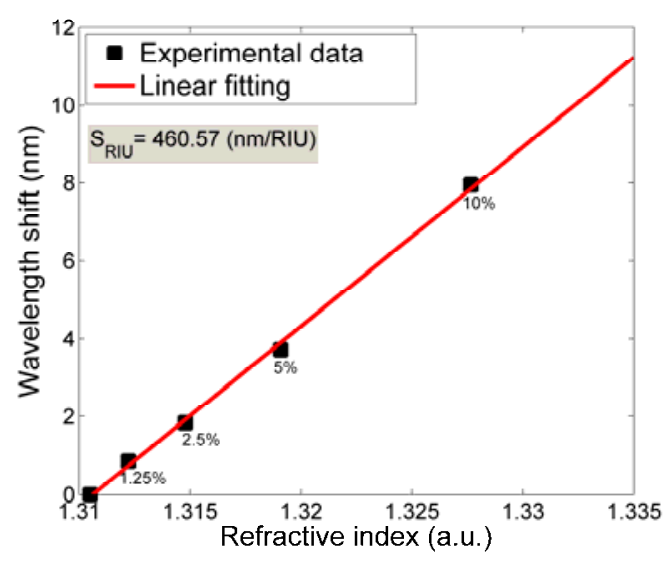

(b)
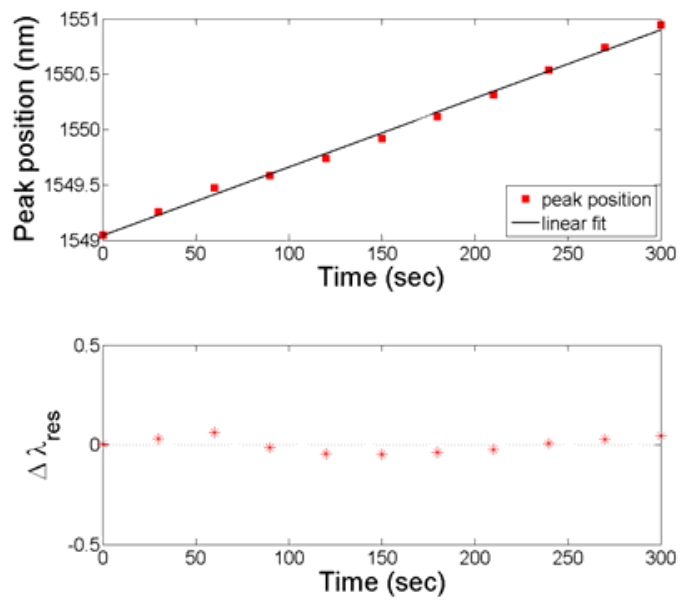

(d)

Fig. 3: a) Scanning electron microscope picture of the fabricated microdisk sensor. b) Experimental data and linear fit of the wavelength shift due to different NaCl-concentrations. c) Transmission spectra due to evaporation of a liquid film and an increase in $\mathrm{NaCl}$ concentration over time. d) Resonance peak shift over time according to figure c) and deviation of the measurement results from the ideal linear fit.

that low-loss hydrogenated amorphous silicon material can be applied in a variety of different lab-on-chip sensing areas, including chemical, medical and bio-sensing applications.

\section{References}

[1] S. Janz, A. Densmore, D.-X. Xu, P. Waldron, J. Lapointe, J. H. Schmid, T. Mischki, G. Lopinski, A. Delage, R. McKinnon, P. Cheben, and B. Lamontagne, "Silicon photonic wire waveguide sensors," in Advanced Photonic Structures for Photonic and Chemical Detection, X. Fan, ed. (Springer, 2009), pp. 229-264.

[2] A. Harke, M. Krause, and J. Müller, "Low-loss singlemode amorphous silicon waveguides", Electron. Lett. 41, 1377-1379, (2005).

[3] T. Lipka, O. Horn, J. Amthor, J. Müller, "Low-loss multilayer compatible a-Si:H optical thin films for photonic applications", J. Europ. Opt. Soc. Rap. Public. 12033 Vol 7, (2012).
[4] F.Vollmer, and L. Yang, "Label-free detection with high- $Q$ microcavities: a review of biosensing mechanisms for integrated devices," in Nanophotonics 1, pp.267-291, De Gruyter, (2012).

[5] F. Dell'Olio, and V. Passaro, "Optical sensing by optimized silicon slot waveguides," Opt. Express 15, 4977-4993 (2007).

[6] K. F. Palmer, and D. William, "Optical properties of water in near-infrared," Journal of the Optical Society of America, 64(8):1107-1110, (1974).

[7] T. Lipka, J. Amthor, C. Krückel, J. Müller, "High Q-factor hydrogenated amorphous silicon microdisk resonators," Opto-Electronics and Communications Conference (OECC), pp.873874, 2-6 July (2012).

[8] H. Su and X. G. Huang, "Fresnel-reflection-based fiber sensor for on-line measurement of solute concentration in solutions", Sensors and Actuators B-Chemical, 126:579-582, (2007). 